\title{
LVDT Conditioning on the LHC Collimators
}

\author{
Alessandro Masi, Arnaud Brielmann, Roberto Losito, and Michele Martino
}

\begin{abstract}
The Position Readout and Survey system of the LHC collimators has to monitor in real time (RT), at a frequency close to $100 \mathrm{~Hz}$, the position of the 555 synchronized axes with an accuracy of $20 \mu \mathrm{m}$. Radiation hard Linear Variable Differential Transformers (LVDT) are used as absolute position sensors for their ruggedness, intrinsic accuracy and infinite resolution. Because of the radioactive environment the conditioning electronics is located in safe zones up to $800 \mathrm{~m}$ away from the sensors. Under these conditions, standard conditioning techniques cannot guarantee the accuracy requested by this application because of cable impedance, noise immunity, crosstalk between signals of different sensors passing within the same multiwire cable and temperature stability. A fully digital approach based on a sine-fit algorithm has been followed and will be described showing the excellent results measured up to now. Particular attention is devoted to the RT implementation of the algorithm on the PXI platform from National Instruments.
\end{abstract}

Index Terms-Digital signal processing (DSP), linear variable differential transformers (LVDT), PXI, sine fit.

\section{INTRODUCTION}

$\mathbf{T}$ HE high luminosity performance of the LHC relies on storing, accelerating, and colliding beams with unprecedented intensities. The energy density of the nominal beam is 1000 times higher than previously achieved in proton storage rings. Tiny fractions of the stored beam suffice to quench a super-conducting LHC magnet or even to destroy parts of the accelerator. More than 100 collimators will therefore be installed to protect the machine from uncontrolled particle losses, and to absorb energetic particles outside the nominal beam core [1].

An LHC collimator consists of two 1-meter long blocks that can be made of different materials (graphite, copper, tungsten) depending on their position in the lattice.

Each jaw can be moved on both ends by stepping motors that can position it at a well defined angle and distance with respect to the nominal beam trajectory. The required accuracy of positioning is one tenth of the beam core diameter, which at nominal energy will be of the order of $200 \mu \mathrm{m}$. We therefore assumed as project parameter for the accuracy $20 \mu \mathrm{m}$.

The stepping motors are controlled in open loop, in the sense that they follow predefined trajectories without feedback from a position sensor. The trajectories are sent to the low level control system by a Central Supervisory System (CSS) application coordinating all the collimators [2]. The concept of open loop control is based on the precision of the cinematic chain that has been designed to be free of mechanical play. Every collimator is

Manuscript received May 10, 2007; revised November 20, 2007.

A. Masi, A. Brielmann, and R. Losito are with CERN, Geneva 1211, Switzerland (e-mail: alessandro.masi@cern.ch).

M. Martino is with the Dipartimento di Scienze Fisiche, Universita di Napoli Federico II, 80126 Napoli, Italy.

Digital Object Identifier 10.1109/TNS.2007.913473 tested before installation to verify that the mechanical play and the non linearity of positioning remain below $20 \mu \mathrm{m}$ on every axis.

However in order to ensure the level of machine safety required by the LHC, an independent Position Readout and Survey (PRS) module has been defined in the low level control system to verify in real time the actual position of the jaws. Depending on the type and energy of the circulating beams, the PRS will be enabled to trigger a beam abort in case of discrepancy between the desired and the measured position of the jaw.

The PRS is based on radiation tolerant linear LVDTs specifically designed and built for this project by Measurement Specialties Inc. On each collimator up to seven LVDTs have to be monitored, four of which will measure the absolute position of each jaw's end, one the collimator's vertical position. The two remaining sensors will measure the distance between the jaws to build a certain level of redundancy and fault tolerance in the system.

Each PRS will control up to three collimators. Overall, the PRS systems will have to monitor in real time more than 700 LVDTs. All the PRS systems have to be synchronized to a trigger coming from the supervisory collimator control system at the start of a motion profile.

With the nominal motor speed of 400 steps per second, and a reduction factor of $2 \mathrm{~mm} /$ revolution, a survey frequency of $100 \mathrm{~Hz}$ is required to detect position errors not exceeding $20 \mu \mathrm{m}$.

The most challenging problem in ensuring the required accuracy is given by the environmental conditions of the LHC. Due to the high level of radiation expected in the proximity of the collimators (several MGrays/year), no electronics can be embedded in the sensors or in the motors. On top of that, radiation safe alcoves for the installation of the driving electronics can be up to $1 \mathrm{~km}$ far from the collimator itself, generating a complex matching problem for the conditioning electronics. The impedance of the cable can in fact transform on long distances the impedance of the sensor from inductive to capacitive. Standard off the shelf conditioners do not generally provide a wide range of tuning to cover cable distances from $20 \mathrm{~m}$ up to $800 \mathrm{~m}$ as required for the LHC collimators, while preserving a satisfactory stability and accuracy of positioning. In Sections II-V we will review the state of the art in LVDT conditioning techniques, and put in evidence the pros and cons of the different approaches.

I will then detail our original approach that allowed us at the same time to cope with long cables and to feed up to seven sensors with a single multiwire cable without interference from one sensor to the other, allowing considerable savings on cabling. I will show then the implementation of the algorithm on a real time platform, and provide figures about accuracy and time response of the measurements. 


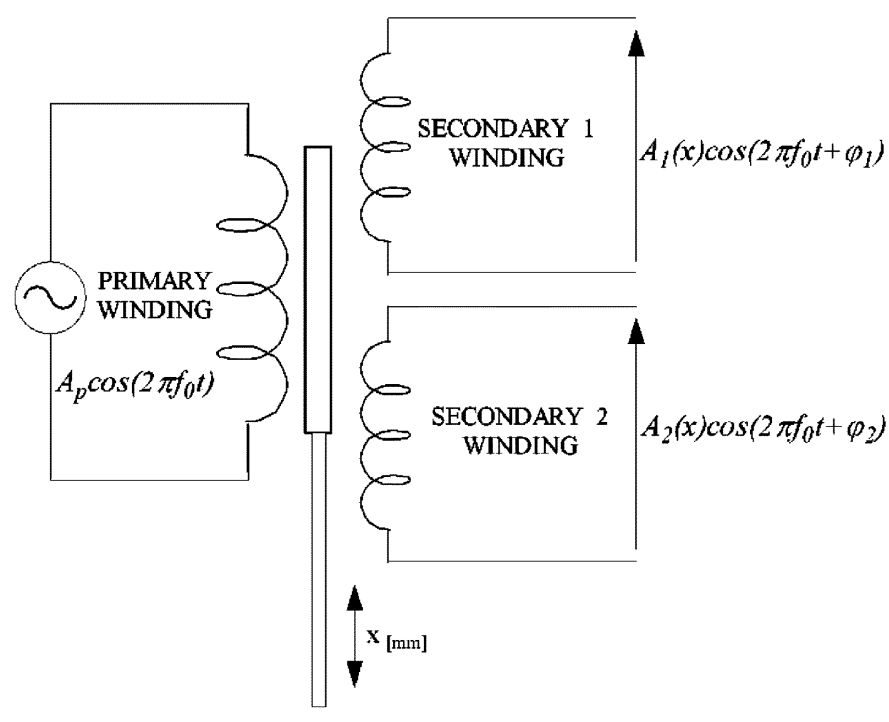

Fig. 1. Working principle of the LVDT.

\section{THE LiNEAR VARIABLE DifFERENTIAL TRANSFORMER: CONDITIONING TECHNIQUES}

\section{A. The LVDT Working Principle}

The linear variable differential transformer (LVDT) has been chosen as displacement sensor for the LHC collimator jaws because of its good characteristics in terms of linearity, resolution, repeatability, and reliability. Special radiation hard (50 MGrays) LVDTs have been designed to have a linearity error below $0.2 \%$. Being based on a contactless construction, they will provide a longer lifetime and a high repeatability. The LVDT principle is shown in Fig. 1. By applying a voltage sinewave to the primary winding, a voltage will be induced in the secondaries. The difference between the two secondary voltages will depend linearly on the displacement of the central moveable core.

If we call $s(t)$ the LVDT feeding signal at frequency $f_{0}$ :

$$
s(t)=A_{P} \cos \left(2 \pi f_{0} t\right) .
$$

The two secondary signals can be expressed as:

$$
\begin{aligned}
& y_{1}(t)=A_{1}(x) \cos \left(2 \pi f_{0} t+\varphi_{1}\right) \\
& y_{2}(t)=A_{2}(x) \cos \left(2 \pi f_{0} t+\varphi_{2}\right) .
\end{aligned}
$$

Where the amplitudes of $y_{1}$ and $y_{2}$ depend on the LVDT sensitivity and on the position of the LVDT core.

\section{B. State of the Art in Conditioning Techniques}

The LVDT is generally used either in a 4-wire or in a 5-wire configuration.

In the 4-wire configuration the signal at the output terminals is the difference of the secondary windings. The 4-wire LVDT does not have a center tap available. As the magnetically permeable core is moved from the center position, the magnetic coupling between the primary and one secondary coil increases, while the other simultaneously decreases. Therefore, the difference between the two output signals is a non-zero sine wave with amplitude proportional to the core position.
The 5-wire configuration includes an additional wire which represents the connection point (center tap) for both secondary windings, allowing the individual measurement of the voltage at each secondary winding with respect to the center tap.

The traditional technique of LVDT signal processing consists in a synchronous demodulation of the differential secondary voltage with respect to the primary [3]. The effect of noise with frequency components far from the frequency of the sinewave sent to the primary is reduced. Fluctuations of the primary signal amplitude are also eliminated at the first order. Nevertheless, most LVDTs present a phase difference between the signal applied to the primary and the signal resulting from the difference between the two secondaries. The angle depends on the operating point.

The differential secondary voltage can be expressed as:

$$
y(t)=A(x) \cos \left(2 \pi f_{0} t+\varphi\right)
$$

where $A(x)$ depends on the LVDT core position.

The synchronous demodulation is obtained by multiplying a signal synchronous with the carrier $s(t)$ and low pass filtering:

$$
\begin{aligned}
& {\left[y(t) \cdot \cos \left(2 \pi f_{0} t\right)\right]_{\text {lowpass }}} \\
& \quad=\left[A(x) \cdot \cos \left(2 \pi f_{0} t+\varphi\right) \cdot \cos \left(2 \pi f_{0} t\right)\right]_{\text {lowpass }} \\
& \quad=\left[A(x) \cdot \frac{\cos (\varphi)}{2}+A(x) \cdot \frac{\cos \left(2 \pi 2 f_{0} t+\varphi\right)}{2}\right]_{\text {lowpass }} \\
& \quad=A(x) \cdot \frac{\cos (\varphi)}{2}
\end{aligned}
$$

As a result the LVDT position depends on the phase difference $\varphi$ which in turn depends on the cable length used to drive the sensor. A manual tuning is therefore needed at installation to compensate the phase error related to different cable lengths. This is the case for instance of the integrated circuit AD698 from Analog Device [4].

Some digital techniques have been developed to estimate with a PLL the phase error in order to correct it automatically [5]. This approach provides a greater accuracy, but they maintain the same original dependence on $\cos (\varphi)$. Near the null point, say for measurement below $5 \%$ of the Full Scale (FS), the variability of $\varphi$ increases and moreover, since the differential secondary signal is very low, it will be heavily affected by noise, at the point that phase recovery may become impossible. In addition in our application the long cables between sensors and electronics transform the impedance seen by the conditioner from inductive to capacitive. In this situation phase recovery would require a very sophisticated algorithm.

The AD598 IC performs an asynchronous demodulation to correct the effect of uncertainty in the null position [6]. It works with a 5-wire configuration and is based on ratiometric conditioning dividing the difference of the LVDT secondary voltages by their sum. This ensures a compensation of the amplitude drift on the excitation signal. The noise immunity of this solution is not better than that obtained with a synchronous demodulation especially if it is used in a 4-wire configuration [7]. Ratiometric conditioning however implies that the sum of the two secondaries has to be independent of the position of the LVDT core to not affect the measurement linearity. 
Spectral techniques, for instance the one based on Goertzel algorithm [8], are characterized by a higher noise immunity but suffer of the spectral leakage problem. A window on the sampled signal has to be applied in the frequency domain to reduce this error.

\section{The Newly Proposed Conditioning Technique}

None of the techniques mentioned above were fully satisfactory for our purposes. The requirements on accuracy and speed of acquisition imposed to adopt a technique highly immune to external noise. Moreover, the wide range of cable lengths from the sensor to the electronics ( $25 \mathrm{~m}$ up to $800 \mathrm{~m}$ ) imposed to have a technique that allows a wide range of tuning without the need of local intervention. We proposed to use a SineFit algorithm [9] to reconstruct the response from the LVDT secondaries and then use the ratiometric formula (5) to compute the absolute position. The details are below.

\section{A. Ratiometric Conditioning}

The position reading of the LVDT is based on a ratiometric decoding of the two secondary voltages. The ratio is given by:

$$
r(x)=\frac{A_{1}(x)-A_{2}(x)}{A_{1}(x)+A_{2}(x)} .
$$

The design of the LHC collimator LVDTs guarantees a constant sum $\left(A_{1}+A_{2}\right)$ over the useful positions range $( \pm 40 \mathrm{~mm})$.

The amplitude demodulation has been performed with a sine fit algorithm [9] because it is an asynchronous demodulation so it does not suffer of the phase error problem. As we will see in the following, no filtering or windowing is required to eliminate the spectral leakage. This algorithm is characterized by high noise immunity and a frequency response that can significantly reduce the crosstalk due to the proximity of different LVDTs.

\section{B. Sine Fit Algorithm Formalization}

The best estimate of the amplitude $A$, the phase $\psi$, and the offset $O$ of a sine wave signal of frequency $f_{0}$ from $N$ of its samples $y_{1} y_{2} \ldots y_{N}$ acquired at the time instants $t_{1} t_{2} \ldots t_{N}$ is obtained minimizing the following error quantity

$$
e=\sqrt{\sum_{i=1}^{N}\left[y_{i}-A_{c} \cos \left(2 \pi f_{0} t_{i}\right)-A_{s} \sin \left(2 \pi f_{0} t_{i}\right)-O_{0}\right]^{2}}
$$

where $K, \psi, O$ are given by:

$$
\left\{\begin{array}{l}
A=\sqrt{A_{c}^{2}+A_{s}^{2}} \\
\psi=\arctan \left(A_{s}, A_{c}\right) \\
O=O_{0}
\end{array}\right.
$$

The square of the error can alternatively be expressed by the matrix equation:

$$
e^{2}\left(\mathbf{x}_{\mathbf{0}}\right)=\left(\mathbf{y}-\mathbf{D}_{\mathbf{0}} \mathbf{x}_{\mathbf{0}}\right)^{\mathbf{T}}\left(\mathbf{y}-\mathbf{D}_{\mathbf{0}} \mathbf{x}_{\mathbf{0}}\right),
$$

where:

$$
\begin{aligned}
\mathbf{y} & =\left[\begin{array}{c}
y_{1} \\
y_{2} \\
\vdots \\
y_{N}
\end{array}\right], \quad \mathbf{x}_{\mathbf{0}}=\left[\begin{array}{c}
A_{c} \\
A_{s} \\
O_{0}
\end{array}\right], \\
\mathbf{D}_{\mathbf{0}} & =\left[\begin{array}{ccc}
\cos \left(2 \pi f_{0} t_{1}\right) & \sin \left(2 \pi f_{0} t_{1}\right) & 1 \\
\cos \left(2 \pi f_{0} t_{2}\right) & \sin \left(2 \pi f_{0} t_{2}\right) & 1 \\
\vdots & \vdots & \vdots \\
\cos \left(2 \pi f_{0} t_{N}\right) & \sin \left(2 \pi f_{0} t_{N}\right) & 1
\end{array}\right] .
\end{aligned}
$$

It can be demonstrated that the minimum of the error function is given by the following equation [9], [10], [11]:

$$
\tilde{\mathbf{x}}_{\mathbf{0}}=\left(\mathbf{D}_{\mathbf{0}}^{\mathbf{T}} \mathbf{D}_{\mathbf{0}}\right)^{-\mathbf{1}} \mathbf{D}_{\mathbf{0}}^{\mathbf{T}} \mathbf{y}=\mathbf{D}_{\mathbf{0}}^{\dagger} \mathbf{y}
$$

where

$$
\mathbf{D}_{0}^{\dagger}=\left(\mathbf{D}_{0}^{\mathbf{T}} \mathbf{D}_{0}\right)^{-\mathbf{1}} \mathbf{D}_{\mathbf{0}}^{\mathbf{T}} .
$$

Estimating the amplitude, phase and offset of a sine wave starting from $N$ samples reduces to computing the elements of the matrix $\mathbf{D}_{0}$, computing the matrix (11) and then multiplying by the vector of the samples.

\section{Algorithm Characterization}

Since one of the biggest concerns is noise immunity, intensive simulations have been carried on with Matlab, to fully characterize the SineFit algorithm in terms of its immunity to different kind of perturbations expected in the operating environment of the LVDT in the LHC.

1) Immunity to White Noise: We first combined a white gaussian noise to the sinusoidal signal. We evaluated the uncertainty on the amplitude estimate for different values of the Signal to Noise Ratio and number of samples acquired at the sampling frequency $f_{s}$.

In Fig. 2 the standard deviation on 500 repetitions is shown as a function of the SNR and at a different number of acquired samples is shown.

An exponential model has been used to fit the standard deviations on the SNR:

$$
\sigma(\mathrm{SNR})=\alpha e^{\beta \cdot \mathrm{SNR}} .
$$

Then the values of the parameters $\alpha$ and $\beta$ have been fitted on the number of samples $N$ with the following model:

$$
\begin{aligned}
& \alpha(N)=a e^{b \cdot N} \\
& \beta(N)=\bar{\beta} .
\end{aligned}
$$

Fig. 3 shows that, for the case $f_{s}=250 \mathrm{kS} / \mathrm{s}$, increasing $N$ from 500 to 2500 the coefficient $\alpha$ is reduced only by a factor of two. Increasing even more substantially the number of points will be less and less beneficial to the noise figure of the algorithm.

$\beta$ on the contrary stays more or less constant. 


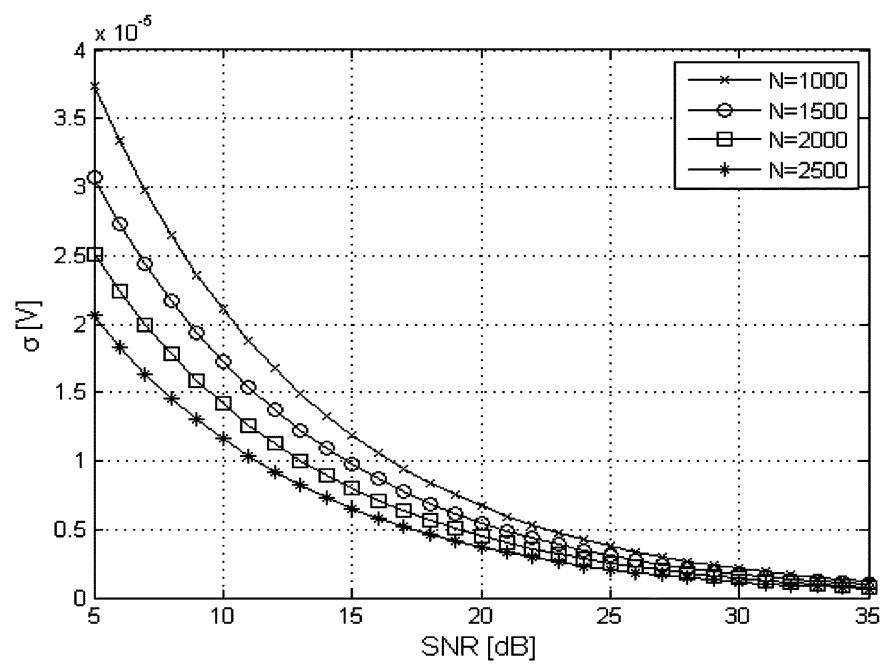

Fig. 2. Amplitude estimate uncertainty on 500 repetitions of a sinusoidal signal with white noise. The simulations refer to different SNR values and number of samples acquired $N$.
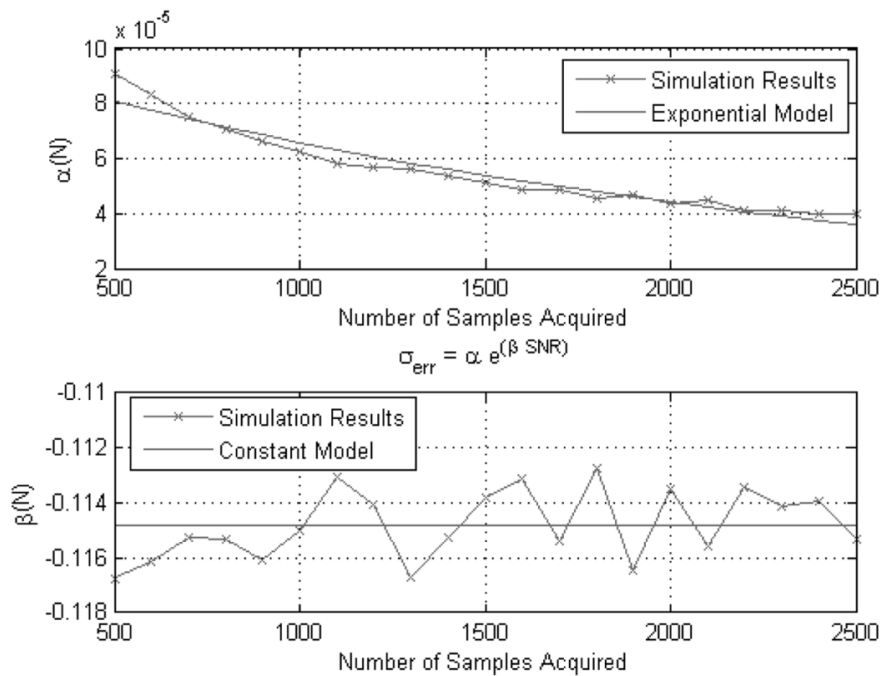

Fig. 3. Fit of the model introduced in (13) to describe the amplitude estimate uncertainty with the sine-fit algorithm on a sinusoidal signal at $f_{0}=2500 \mathrm{~Hz}$, $f_{s}=250 \mathrm{kS} / \mathrm{s}$ with superimposed white noise.

The simulation results can be summarized by the following approximated formulas:

$$
\begin{aligned}
\sigma(N, \mathrm{SNR}) & =9.88 \times 10^{-5} e^{-\left(3.98 \times 10^{-4} N+0.115 \mathrm{SNR}\right),} \\
f_{s} & =250 \frac{\mathrm{kS}}{\mathrm{s}} \\
\sigma(N, \mathrm{SNR}) & =5.83 \times 10^{-5} e^{-\left(2.36 \times 10^{-4} N+0.115 \mathrm{SNR}\right),} \\
f_{s} & =500 \frac{\mathrm{kS}}{\mathrm{s}} .
\end{aligned}
$$

Taking into account the previous simulations, we decided to implement the algorithm with 2000 samples. This number guarantees an uncertainty of less than $10 \mathrm{ppm}$ on the estimate of the amplitude even with the unrealistic case of $15 \mathrm{~dB}$ of SNR. With $250 \mathrm{kS} / \mathrm{s}$ the $N$ samples are acquired in $8 \mathrm{~ms}$. This value is compatible both with the survey frequency requirement and with the position signal dynamics.

2) Immunity to Crosstalk: In order to save on cablings (more than $200 \mathrm{~km}$ of cables have already been pulled in the LHC

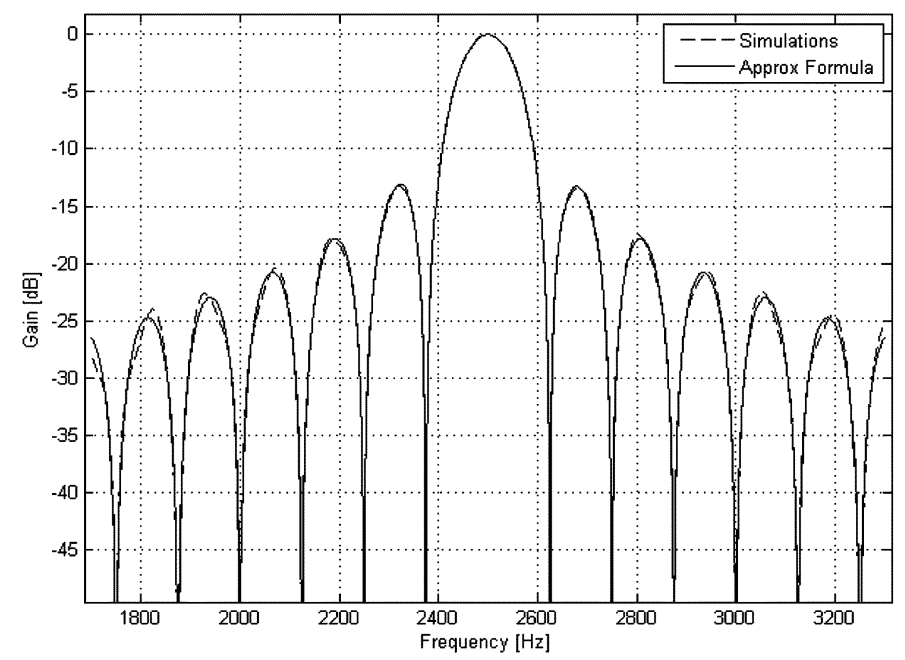

Fig. 4. Frequency response of the Sine Fit algorithm calculated with $f_{0}=$ $2500 \mathrm{~Hz}, N=2000, f_{s}=250 \mathrm{kS} / \mathrm{s}$ SineFit algorithm.

for the collimation project), the seven LVDTs of a single collimator are all connected to the conditioning electronics through the same multiwire cable. A cross talk between different channels can be expected. The frequency response of the SineFit algorithm has been characterized in order to evaluate the influence of a sinusoidal perturbing signal on the amplitude estimate.

The algorithm performs an average of the instantaneous amplitude over the acquisition period (i.e., the time needed to acquire the $N$ samples of the signal, $8 \mathrm{~ms}$ in our case). Let $y(t)$ be the generic sine tone with variable amplitude described by

$$
y(t)=A(t) \sin \left(2 \pi f_{0} t\right) .
$$

Under the assumption that $A(t)$ varies slowly with respect to $f_{0}$, at the survey time $t_{k}^{s}$ the algorithm will return the value expressed in (16):

$$
\tilde{A}\left(t_{k}^{s}\right) \cong \frac{f_{S}}{N} \int_{t_{k}^{s}-N / f_{S}}^{t_{k}^{s}} A(t) d t .
$$

The frequency response has been determined evaluating the amplitude estimated by the SineFit algorithm of a unity sine wave input signal at an arbitrary frequency $f=f_{0}+\Delta f$ expressed as:

$$
\begin{aligned}
u(t) & =\sin (2 \pi f t) \\
& =\sin (2 \pi \Delta f t) \cos \left(2 \pi f_{0} t\right)+\cos (2 \pi \Delta f t) \sin \left(2 \pi f_{0} t\right) .
\end{aligned}
$$

By means of (16) it follows:

$$
\tilde{A}(f) \cong \sqrt{\left(\frac{f_{S}}{\pi N \Delta f} \sin \left(\frac{\pi N \Delta f}{f_{S}}\right)\right)^{2}}=\left|\operatorname{sinc}\left(\frac{\Delta f N}{f_{S}}\right)\right| .
$$

A direct simulation with Matlab matches very well the expression obtained analytically.

Fig. 4 shows clearly that it is possible to find a set of frequencies that do not interfere to each other. Let's introduce

$$
\Delta f_{\text {zero }}=\frac{f_{S}}{N} \text {. }
$$


A tone centered on a frequency spaced by an integer number of $\Delta f_{\text {zero }}$ from the design frequency $f_{0}$ will not perturb the estimate of the $f_{0}$ tone amplitude. In order to ensure an a priori orthogonality of the LVDTs channels, 7 non-interfering (i.e., orthogonal) frequencies have been chosen to excite the LVDTs of the same collimator and 7 different SineFit demodulations are performed.

\section{The Position ReAdout ANd SURVEy System}

\section{A. General Description}

The PRS is responsible for checking the coherence of actual jaws' positions of up to three collimators with those expected by the CSS. To increase the overall reliability of the control system, the PRS is required to be independent from the MDC (Motor Drive Control), the low level control module that directly controls the movement of the stepping motors by sending the STEP and DIRECTION signals to their power drivers, and by reading the angular position of the motors from resolvers mounted on their axes, in order to quickly detect lost steps. To make it independent, the PRS runs on a different machine than the MDC.

Two National Instruments PXI chassis with two different controllers are therefore attached to one, two or three collimators and host one the MDC and the other the PRS. The two PXI chassis are completely independent, and do not directly communicate with each other. DIM servers installed on each of them provide connectivity to the CERN middleware CMW and exchange commands and data with the CSS through the CERN framework FESA (Front End Software Architecture).

The PRS monitors the position of the jaws through the up to seven LVDTs installed on each collimator at a rate of up to $100 \mathrm{~Hz}$. Such a high rate is necessary to ensure that the relative position of two jaws in different collimators remains unchanged during the execution of a movement function within a tolerance of $20 \mu \mathrm{m}$. The maximum speed of the motor will in fact be of one revolution per second, for a linear movement of $2 \mathrm{~mm} /$ revolution.

Measurements in a laboratory with metrology palmers have shown that the reading from the PRS, once each LVDT has been properly calibrated, is accurate to within less than $10 \mu \mathrm{m}$ at normal pressure and temperature even with cable lengths up to $800 \mathrm{~m}$. In addition, the sensors themselves have been designed to have a negligible thermal sensitivity. Temperature changes up to $30 \mathrm{~K}$ (well above what is expected on a LVDT in the LHC) produce a measurement drift below $10 \mu \mathrm{m}$.

The PRS fulfills then the requirements on accuracy as a result of the excellent noise rejection and independence from the sensor non linearity of the sine fit algorithm combined to ratiometric conditioning, as well as of the careful design of the sensor and of the collimator mechanical parts. The fulfillment of the requirements on the time response is the merit of the real time architecture used and of the RT optimization of the conditioning algorithm combined with the performance of the real time platform chosen for the implementation, the National Instruments PXI.

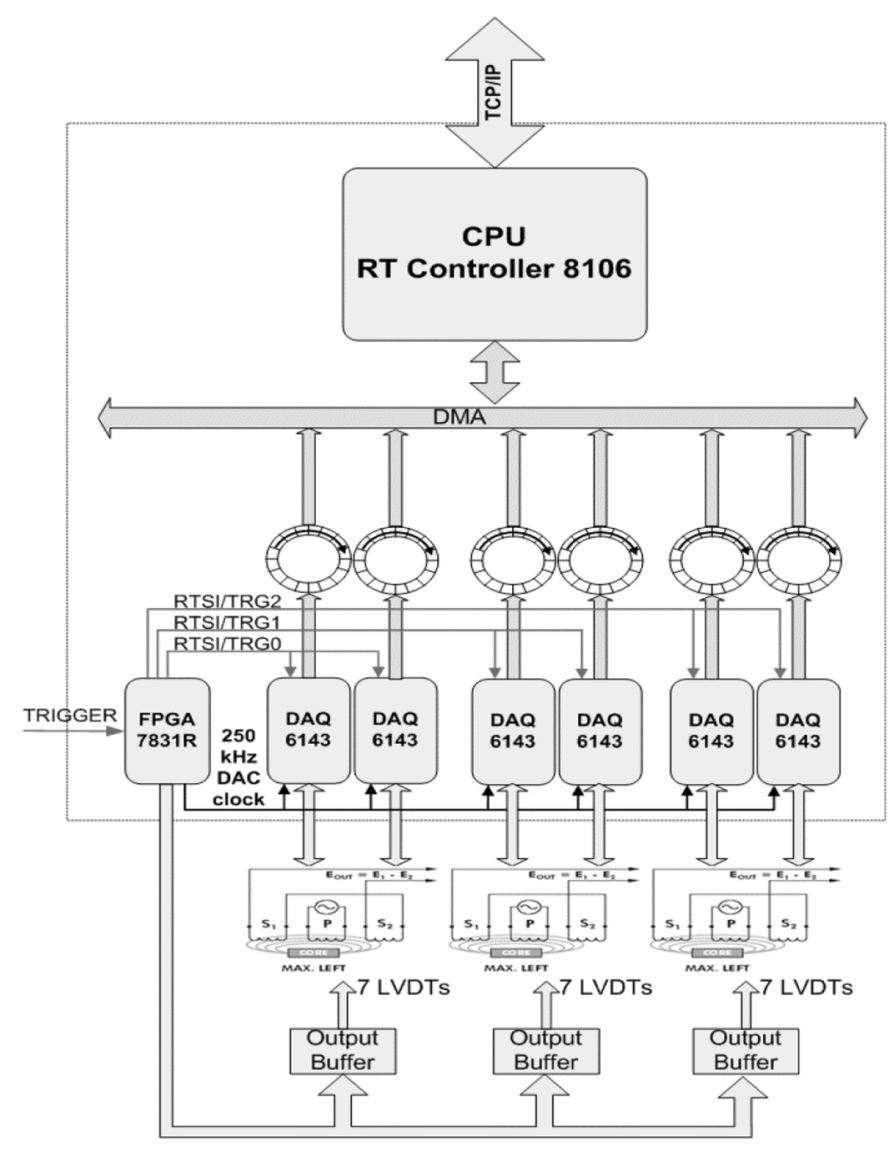

Fig. 5. Hardware Architecture of a PRS.

\section{B. The Hardware Architecture}

The hardware architecture of a PRS for 3 collimators has already been described in details in [2]. The description is here summarized for convenience, and shown in Fig. 5.

A National Instruments PXI chassis is equipped with an RT controller NI-PXI 8106 based on an Intel ${ }^{\circledR}$ Core 2 Duo Processor at $2.16 \mathrm{GHz}$.

For each collimator controlled by the specific chassis, two DAQ cards NI PXI 6143 with 8 simultaneous differential analog inputs sampled at 16 bit and $250 \mathrm{kS} / \mathrm{s}$ are used to acquire the 14 signals coming from the secondary coils of the 7 LVDTs installed on each collimator.

Data acquired are transferred to the CPU via DMA using circular buffers as shown in Fig. 5. In this way the acquisition of the signals coming from the LVDT secondaries is decoupled from their processing, releasing CPU resources.

Furthermore, an FPGA card NI PXI 7831R having 8 analog outputs is used to generate the feeding signals for the 21 LVDTs. Seven outputs are used to generate sinewaves of different frequencies, orthogonal with respect to the frequency response of the sine-fit algorithm used.

All the signals sent to or coming from the LVDTs are interfaced through custom PCB in Europe format ${ }^{1}$ to ensure at the same time reliability and modularity.

${ }^{1}$ CEI 60297-3-101, CEI 60297-3-102, CEI 60297-3-103/IEEE 1101.1, IEEE $1101.10 / 11$ 

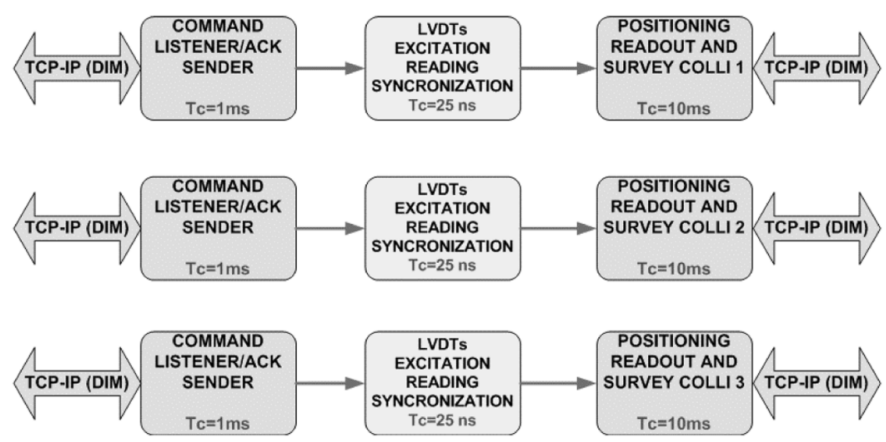

Fig. 6. Software Architecture.

Every signal coming from the analog outputs of the 7831 card is split on three different lines in the Europe cards, and then amplified individually through buffer amplifiers to properly drive each sensor over the long cables. In case of fault on a power stage of one of the collimators, the two others will not be affected.

The same FPGA card is also responsible for the synchronization of all the DAQ cards on the precise $10 \mathrm{MHz}$ clock distributed to all the PXI chassis as well as for the synchronization of the motion survey process for each collimator on the trigger signal sent by the CSS.

A $250 \mathrm{kHz}$ clock for all the DAQ cards is generated by the FPGA from the CLK/10 on the RTSI bus used as reference clock. The start of the readout and survey loops for each collimator is triggered respectively by the signals TRG0, TRG1 and TRG2 on the RTSI bus generated by the FPGA at reception of a trigger from the CSS.

\section{Software Architecture}

1) RT Tasks Architecture: The following functionalities are implemented in the PRS:

- pre-processing the commands received from the CSS: coherence check, protocol check;

- preparation of the upper and lower threshold profiles for the collimator axes and the two gaps;

- feeding the up to 21 LVDTs with signals at proper frequencies;

- reading up to 21 LVDTs with $20 \mu \mathrm{m}$ accuracy at a rate of up to $100 \mathrm{~Hz}$;

- synchronization of the motion profiles survey process on the trigger received by the CSS.

Most of these functions are executed by RT tasks running on the PXI controller NI-PXI 8106, apart from the excitation of the sinusoidal waves to feed the LVDTs and the synchronization task, which are entirely implemented in the FPGA of the NI-PXI 7831 card.

The architecture of the RT tasks executed on the PRS for the readout and survey of three collimators is shown in Fig. 6, where grey tasks are executed on the CPU and light-colored tasks on the FPGA.

The CPU command listener waits for commands or settings sent by the CSS on the related DIM services. The event recognition loop has $1 \mathrm{~ms}$ cycle time and the highest priority to ensure negligible reaction time. At reception of the request of motion profile survey, it prepares upper and lower limit arrays for each axis according to the boundary function sent by the CSS. The limits represent thresholds for triggering a beam abort. A linear interpolation is applied to update the threshold value each $10 \mathrm{~ms}$. An acknowledge is then sent to the CSS to inform it that the PRS is ready for the profile survey and is waiting for the hardware trigger.

The synchronization of the reading and survey process on the CPU is performed by the FPGA card (task LVDTs excitation reading and synchronization). As soon as the trigger is received from the CSS a $100 \mathrm{~Hz}$ clock is generated on the RTSI trigger line related to the collimator to survey. This accurate timing signal synchronizes the CPU task that performs the LVDTs reading and the comparison with the associated thresholds. This approach guarantees that the time jitter on the LVDTs reading will not exceed 100 microseconds, the error on position reading will therefore remain well within specifications.

The position readout and survey task on the CPU performs the processing of the signals of the 7 LVDTs installed on the collimator using a ratiometric conditioning. The time needed to execute a full reading cycle for all the 21 LVDTs of one collimator using 2000 samples for each signal has been measured to be $1 \mathrm{~ms}$ with a jitter below $50 \mu \mathrm{s}$.

2) Optimization for $R T$ Implementation: The frequency $f_{0}$ is known therefore the matrix $D_{0}$ in (9) can be calculated just once per sensor. Furthermore as only the amplitudes of the secondaries are needed to evaluate the sensor reading we do not need to calculate the offset and the matrix $D_{0}$ can be further simplified to an $N \times 2$ instead of an $N \times 3$. For a single secondary channel we have:

$$
\mathbf{D}=\left[\begin{array}{cc}
\cos \left(2 \pi f_{0} t_{1}\right) & \sin \left(2 \pi f_{0} t_{1}\right) \\
\cos \left(2 \pi f_{0} t_{2}\right) & \sin \left(2 \pi f_{0} t_{2}\right) \\
\vdots & \vdots \\
\cos \left(2 \pi f_{0} t_{N}\right) & \sin \left(2 \pi f_{0} t_{N}\right)
\end{array}\right], \quad \mathbf{x}=\left[\begin{array}{c}
A_{c} \\
A_{s}
\end{array}\right]
$$

For $L$ LVDTs an $N \times 2 L$ matrix has to be created and pseudoinverted:

$$
\mathbf{D}_{L}=\left[\begin{array}{lllll}
\mathbf{c}_{f_{1}} & \mathbf{s}_{f_{1}} & \ldots & \mathbf{c}_{f_{L}} & \mathbf{s}_{f_{L}}
\end{array}\right]
$$

where

$$
\mathbf{c}_{f_{j}}=\left[\begin{array}{c}
\cos \left(\frac{2 \pi f_{j}}{f_{s}}\right) \\
\vdots \\
\cos \left(\frac{2 \pi f_{j} N}{f_{s}}\right)
\end{array}\right], \quad \mathbf{s}_{f_{j}}=\left[\begin{array}{c}
\sin \left(\frac{2 \pi f_{j}}{f_{s}}\right) \\
\vdots \\
\sin \left(\frac{2 \pi f_{j} N}{f_{s}}\right)
\end{array}\right] .
$$

The data coming from the acquisition boards and sampled at a frequency $f_{s}$, are stored in an $N \times 2 L$ matrix and then multiplied with the matrix $\mathbf{D}_{L}^{\dagger}$, the pseudo-inverse of the matrix in (21), so at each survey cycle a simple matrix product is performed to produce a $(2 L \times 2 L)$ coefficients' matrix estimate. $2 L$ sinusoidal signals can be in fact expressed by the following equation:

$$
\mathbf{Y}_{L}=\mathbf{D}_{L} \mathbf{X}_{L}
$$




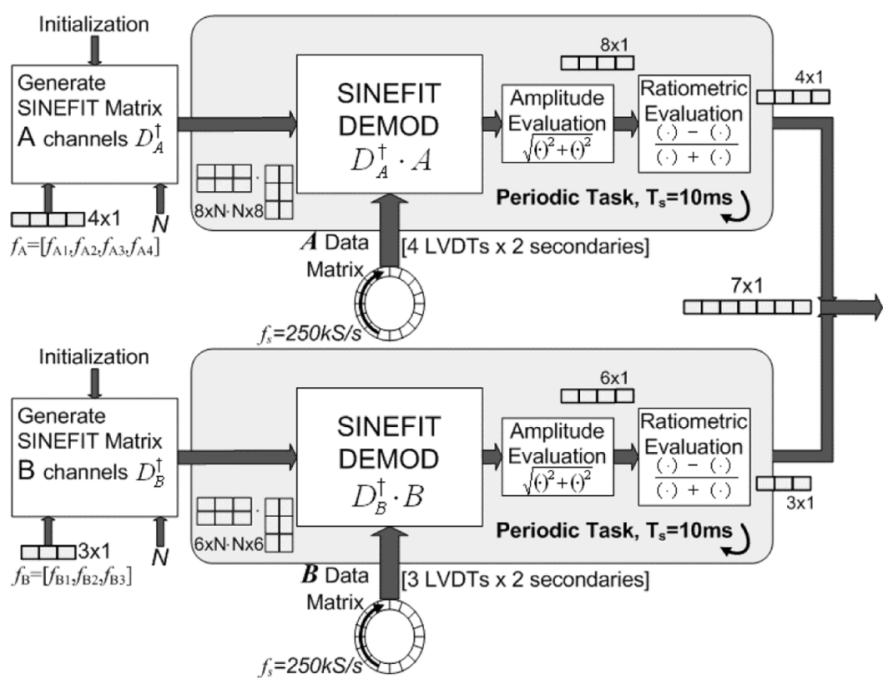

Fig. 7. The Positioning Readout and Survey Task.

in which the amplitudes are:

$$
\begin{aligned}
\mathbf{X}_{L} & =\operatorname{diag}\left\{\mathbf{A}_{f_{1}}^{\left(s_{1}, s_{2}\right)}, \ldots, \mathbf{A}_{f_{L}}^{\left(s_{1}, s_{2}\right)}\right\} \\
\mathbf{A}_{f_{j}}^{\left(s_{1}, s_{2}\right)} & =\left[\begin{array}{ll}
A_{f_{f_{j}}}^{s_{1}} & A_{c_{f_{j}}}^{s_{2}} \\
A_{s_{f_{j}}}^{s_{1}} & A_{s_{f_{j}}}^{s_{2}}
\end{array}\right] .
\end{aligned}
$$

Equation (23) relates $2 L$ ideal sinusoidal signals to their amplitudes, decomposed in cosine and sine components, while (24) describes the diagonal structure of the amplitudes' matrix in which each block, described by (25), gives contributions only for its own frequency. The $s_{1}, s_{2}$ superscripts refer to the first and the second secondary respectively.

Finally the best amplitudes' matrix estimate is given by:

$$
\tilde{\mathbf{X}}_{L}=\mathbf{D}_{L}^{\dagger} \mathbf{Y}_{L}
$$

Two acquisition boards are needed to sample all the LVDTs of a same collimator, respectively 8 channels on board A and 6 channels on board B. The demodulation scheme specified in (21) to (25) has therefore been split: two SineFit matrices are generated at startup pseudo-inverting one matrix for the 4 LVDTs connected to the board A, and one for the 3 LVDTs connected to the board B. At each reading cycle two matrices come from the DMA to be multiplied with the fit matrices. Fig. 7 describes the reading and survey task for a single collimator.

\section{EXPERIMENTAL RESULTS}

\section{A. Software Performances}

Fig. 8 shows the statistics for the parallel readout of 3 collimators' LVDTs running on the same CPU. The execution time with 2000 samples acquired for each channel and at each cycle is around $1 \mathrm{~ms}$ per collimator with a negligible jitter.

\section{B. Single LVDT Reading Characterization}

Some measurements have been carried out to characterize the single LVDT reading performances. The nominal configuration is with $N=2000$ samples and no motor driver running during
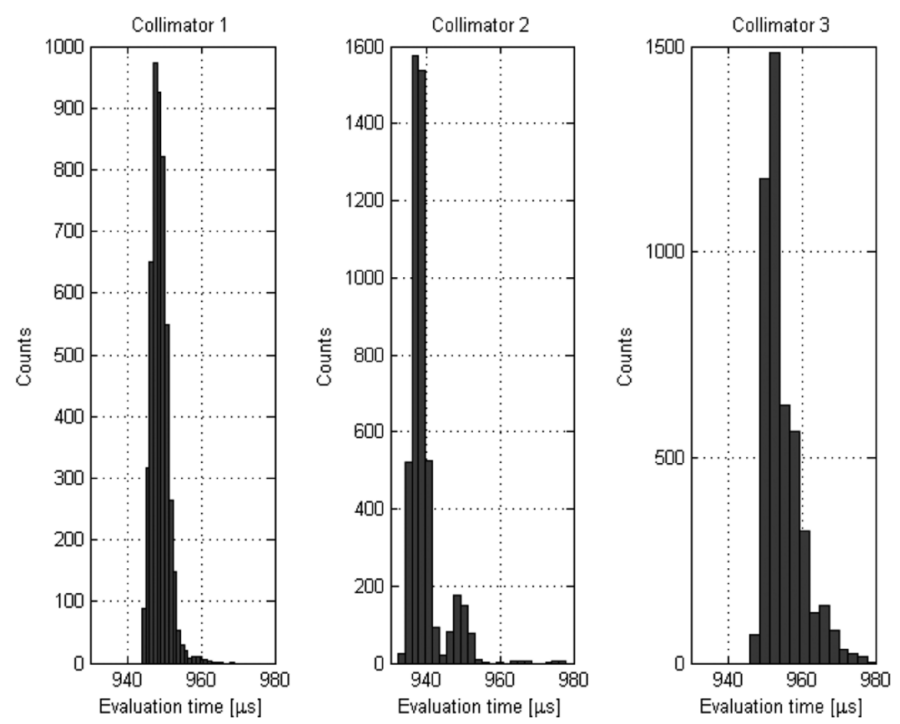

Fig. 8. Each reading task runs in parallel and is completed in approximately 1 ms. No significant differences exist between them and the time jitter is negligible. The statistics are evaluated on 5000 readings each one coming from 2000 samples per channel.
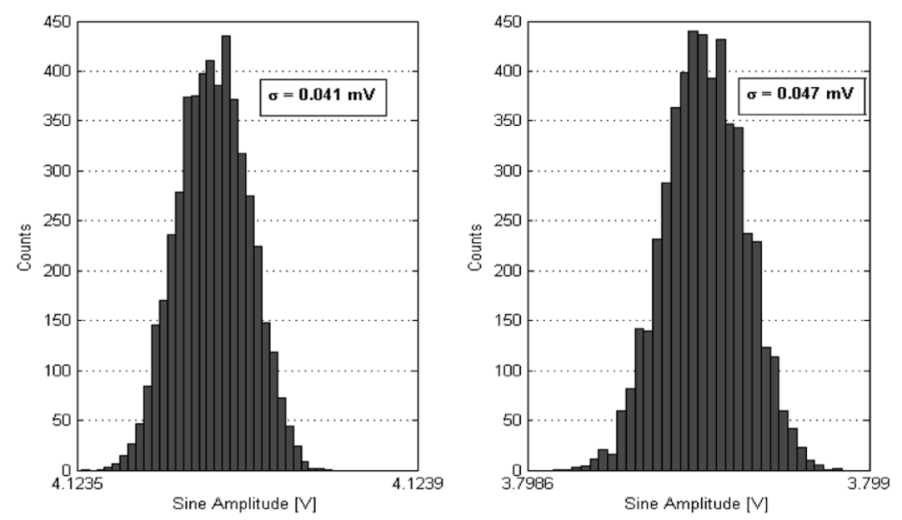

Fig. 9. Typical performance of the channel amplitude estimate. On the left, histogram of the first secondary of LVDT A of Collimator 2 (short cable); on the right the second secondary. $f_{0}=2125 \mathrm{~Hz}$. The statistics are evaluated on 5000 readings.

the test. The nominal gain of $100 \mathrm{~mm} /$ ratiometric unit is assumed for all the LVDTs. Fig. 9 shows the typical amplitude estimate, in the lab setup, for the two secondaries of a single LVDT connected to the PRS by a short cable. The SNR measured on the signals is close to $30 \mathrm{db}$. The standard deviation evaluated on 5000 readings is about $10 \mathrm{ppm}$ of the amplitude. This value is in reasonable agreement with the approximated formulas (14) obtained as simulations result.

To take into account the possibility of noise generated by the stepping motor drivers power electronics we added noise on one secondary of the LVDT using the experimental setup of Fig. 10. We applied square wave noise at $9 \mathrm{kHz}$ and $18 \mathrm{kHz}$, since the LHC motor drivers work at a chopping frequency of $18 \mathrm{kHz}$ and sub-harmonics may be present due to the PWM control; the main sub-harmonic involved is the half order [12]. The square waves have been generated with a SRS DS-360 frequency generator; to add the "noise" to the useful channel a 


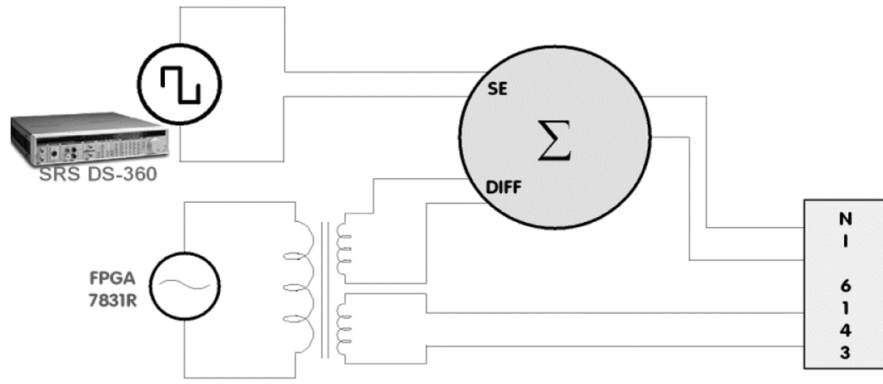

Fig. 10. Measurement setup for motor driver noise immunity test.

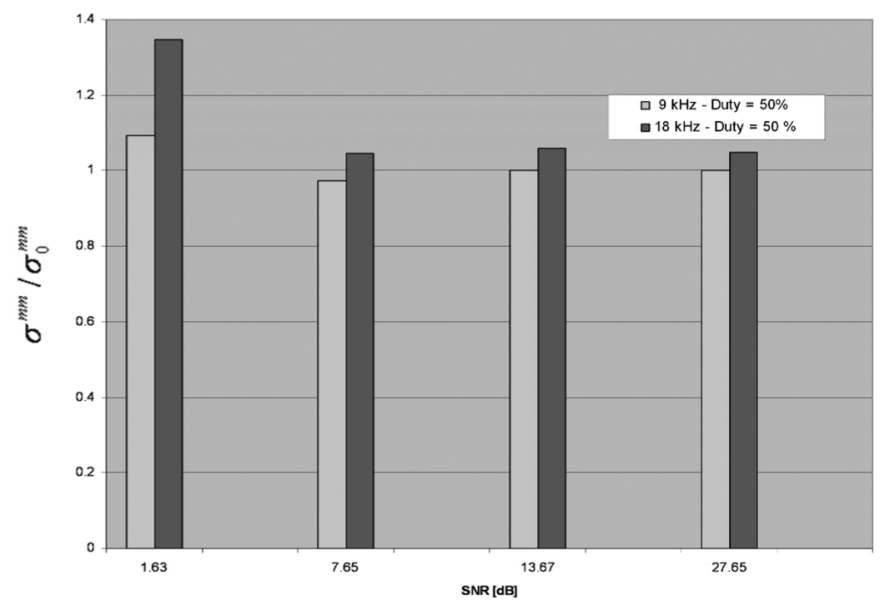

Fig. 11. The position reading $\sigma^{\mathrm{mm}}$ have been normalized to the nominal value $\sigma_{0}^{\mathrm{mm}}$ with no square wave noise applied in order to highlight the effect of the noise applied. Each value plotted is the average of two different measurements, in which the $\sigma^{\mathrm{mm}}$ is evaluated on $N=5000$ readings.

Single Ended-Differential mixer has been built, since the output of the DS-360 is single ended while the LVDT's signals are differential. The ratio between the standard deviation of the position reading at various SNR level and the standard deviation when no noise is applied has been evaluated. The reference standard deviation $\sigma_{0}^{\mathrm{mm}}$ is measured with the mixer connected and the generator switched off.

The experimental results are summarized in Fig. 11. The immunity is very good for all the reasonably expected values of the SNR.

\section{Multiple LVDTs Reading Characterization}

Finally the performance of the overall system has been measured in the nominal conditions. A multiwire $500 \mathrm{~m}$ long cable has been used to connect the 21 LVDTs of 3 collimators to the PRS. Fig. 12 shows the amplitude spectrum of a LVDT secondary channel measured with an Agilent E7405 EMC Analyzer. As expected all the other LVDT's frequencies were detected. The amplitude of the tones is $60 \mathrm{~dB}$ below the main tone, however the effect of these spurious tones is negligible since the frequencies are chosen to be orthogonal.

Fig. 13 shows statistics for some LVDTs connected both with short cable and $500 \mathrm{~m}$ long cable. The difference between the

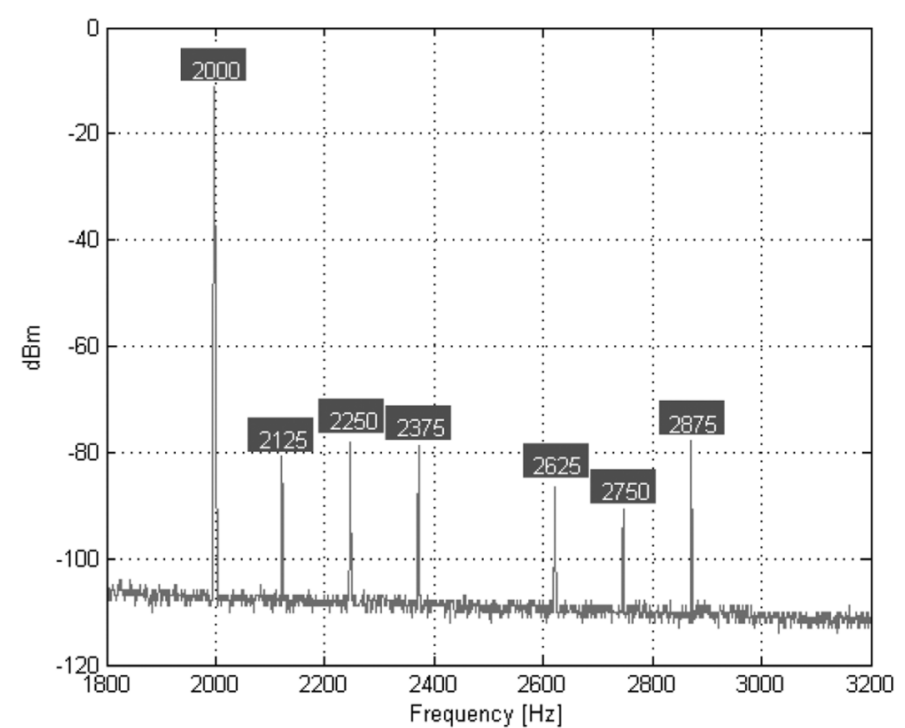

Fig. 12. Typical spectrum of a LVDT secondary channel.
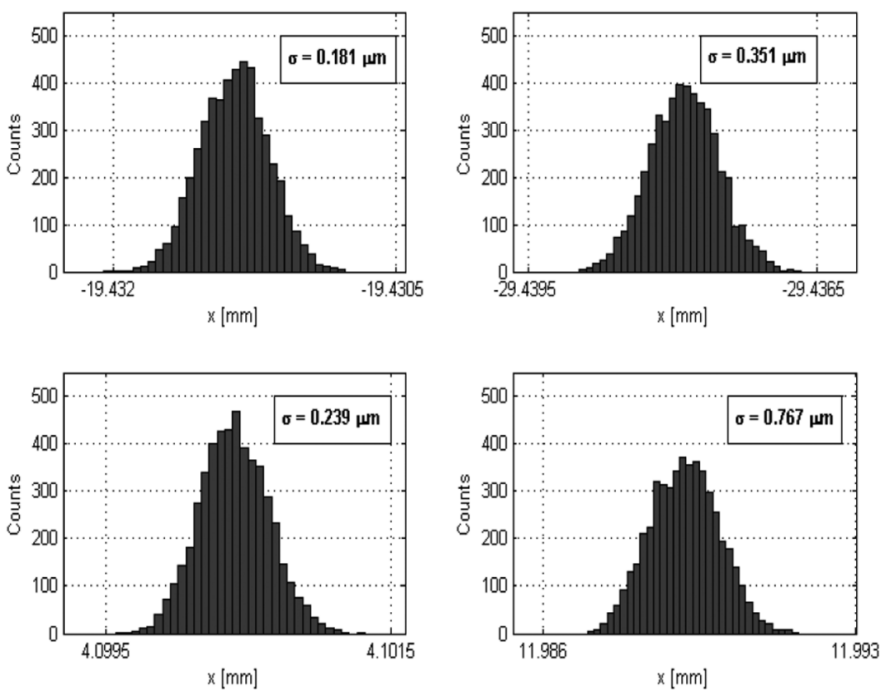

Fig. 13. Position reading performance with the lab setup.Upper. Standard deviations of two LVDTs on 5000 readings with $500 \mathrm{~m}$ long cable.Lower. Standard deviations of two LVDTs on 5000 readings with short cable.

standard deviations can be explained mainly by the different excitation frequencies and, in second place, by the different behavior of the front end channels.

Anyway the standard deviations of the readings are all below $1 \mu \mathrm{m}$ with the laboratory setup and no dramatic degradation can be foreseen in normal operation.

\section{ACKNOWLEDGMENT}

The authors would like to thank J. Lendaro, B. Simon, C. Mitifiot, and C. Serpico for their support on the technical realization of prototypes and measurements. They would also like to thank Prof. F. Cennamo and Prof. V. G. Vaccaro for their useful discussions and suggestions. 


\section{REFERENCES}

[1] R. Assmann et al., "The final collimation system for the LHC," presented at the Euro. Particle Accelerator Conf. (EPAC), Edinburgh, Scotland, U.K., 2006.

[2] A. Masi and R. Losito, "LHC collimators low level control system," IEEE Trans. Nucl. Sci., vol. 55, no. 1, pp. 333-340, Feb. 2008.

[3] J. Szczyrbak and E. D. D. Schmidt, LVDT Signal Conditioning Techniques. London, U.K.: Lucas Varity, 2005.

[4] Universal LVDT Signal Conditioner, Norwood, MA, "AD698 application note analog devices," 1995.

[5] M. F. Ralph, S. W. Robert, and R. L. David, "A DSP-based modified costas receiver for LVDT position sensors," in Proc. 17th IEEE Instrumentation Meas. Technol. Conf., 2000, pp. 1448-1452.

[6] Analog Devices, Norwood, MA, "LVDT signal conditioner," AD598 Appl. Note, 1989.
[7] R. S. Weissbach, D. R. Loker, and R. M. Ford, "Test and comparison of LVDT signal conditioner performance," in Proc. 17th IEEE Instrumentation Meas. Technol. Conf., 2000, pp. 1143-1146.

[8] D. Crescini, A. Flammini, D. Marioli, and A. Taroni, "Application of an FFT-based algorithm to signal, processing of LVDT position sensors," IEEE Trans. Instrum. Meas., vol. 47, no. 11, pp. 1119-1123, Oct. 1998.

[9] IEEE Standard for Terminology and Test Methods for Analog-to-Digital Converters, IEEE Standard 1241, 2000.

[10] E. H. Moore, "On the reciprocal of the general algebraic matrix," Bulletin Amer. Math. Soc., vol. 26, pp. 394-395, 1920.

[11] R. Penrose, "A generalized inverse for matrices," Proc. Cambridge Philosophical Soc., pp. 406-413, 1955.

[12] A. Eisenberg and G. Fedele, "Describing function-based analysis of a class of PWM feedback control systems," in Proc. 25th IASTED Int. Conf., 2006, pp. 49-54. 